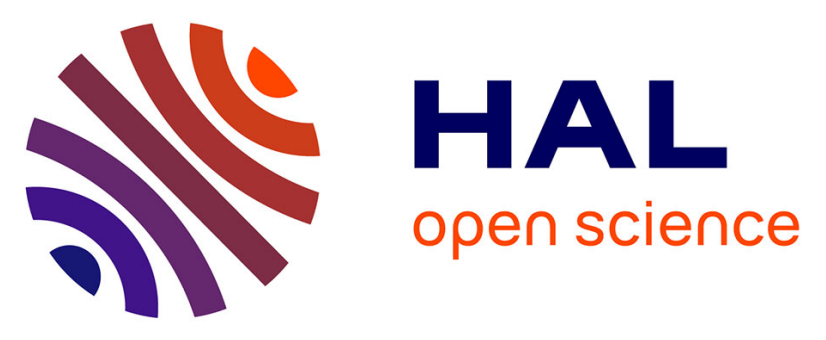

\title{
Time-dependent density functional theory molecular dynamics simulation of doubly charged uracil in gas phase
}

Pablo López-Tarifa, Marie-Anne Hervé Du Penhoat, Rodophe Vuilleumier, Marie-Pierre Gaigeot, Ursula Rothlisbergert, Ivano Tavernelli, Arnaud Le Padellec, Jean-Philippe Champeaux, Manuel Alcamí, Patrick Moretto-Capelle, et al.

\section{- To cite this version:}

Pablo López-Tarifa, Marie-Anne Hervé Du Penhoat, Rodophe Vuilleumier, Marie-Pierre Gaigeot, Ursula Rothlisbergert, et al.. Time-dependent density functional theory molecular dynamics simulation of doubly charged uracil in gas phase. Open Physics, 2014, 12 (2), pp.97-102. 10.2478/s11534-0140428-0 . hal-00982119

\section{HAL Id: hal-00982119 https://hal.science/hal-00982119}

Submitted on 30 Mar 2020

HAL is a multi-disciplinary open access archive for the deposit and dissemination of scientific research documents, whether they are published or not. The documents may come from teaching and research institutions in France or abroad, or from public or private research centers.
L'archive ouverte pluridisciplinaire HAL, est destinée au dépôt et à la diffusion de documents scientifiques de niveau recherche, publiés ou non, émanant des établissements d'enseignement et de recherche français ou étrangers, des laboratoires publics ou privés. 


\title{
Time-dependent density functional theory molecular dynamics simulation of doubly charged uracil in gas phase
}

Research Article

Pablo López-Tarifa ${ }^{1, *}$, Marie-Anne Hervé du Penhoat ${ }^{2}$, Rodophe Vuilleumier ${ }^{3}$, Marie-Pierre Gaigeot ${ }^{4,5}$, Ursula Rothlisberger ${ }^{1}$, Ivano Tavernelli ${ }^{1}$, Arnaud Le Padellec ${ }^{6,7}$, Jean-Philippe Champeaux ${ }^{8,9}$, Manuel Alcamí $^{10}$, Patrick Moretto-Capelle ${ }^{8,9}$, Fernando Martín ${ }^{10,11}$, Marie-Françoise Politis ${ }^{4}$

1 École Polytechnique Fédérale de Lausanne, EPFL SB-ISIC-LCBC-BCH, 1015, Lausanne, Switzerland

2 IMPMC UMR-CNRS 7590, Université Pierre et Marie Curie, 4 place Jussieu, 75005 Paris, France

3 École Normale Supérieure, Département de Chimie, UMR 8640 CNRS-ENS-UPMC, 75005 Paris, France

4 LAMBE, UMR-CNRS 8587, Université d'Evry val d'Essonne, Blvd F. Mitterrand, 91025 Evry, France

5 Institut Universitaire de France, 103 Blvd St Michel, 75005 Paris, France

6 CESR, Université de Toulouse, UPS, 31028 Toulouse Cedex 9, France

7 CNRS, UMR5187, F-31028 Toulouse, France

8 Laboratoire Collisions, Agrégats, Réactivité, Université de Toulouse, UPS, 31062 Toulouse, France

9 CNRS, UMR 5589, F-31062 Toulouse, France

10 Departamento de Qímica, Módulo 13, Universidad Autónoma de Madrid, 28049 Madrid, Spain

11 Instituto Madrileño de Estudios Avanzados en Nanociencia (IMDEA-Nanociencia), Cantoblanco, 28049 Madrid, Spain

\section{Received 09 April 2013; accepted 04 December 2013}

\begin{abstract}
:
We use time-dependent density functional theory and Born-Oppenheimer molecular dynamics methods to investigate the fragmentation of doubly ionized uracil in gas phase. Different initial electronic excited states of the dication are obtained by removing electrons from different inner-shell orbitals of the neutral species. We show that shape- equivalent orbitals lead to very different fragmentation patterns revealing the importance of the intramolecular chemical environment. The results are in good agreement with ionion coincidence measurements of uracil collision with $100 \mathrm{keV}$ protons.
\end{abstract}

PACs (2008): 34.10+x,71.15.Pd,71.15.Qe

Keywords: $\quad$ uracil $\bullet$ proton collision $\bullet$ nonadiabatic dynamics $\bullet$ biological damage

(c) Versita sp. z o.o.

*E-mail: pablo.lopez@epfl.ch 


\section{Introduction}

Ionizing radiations (electro-magnetic radiation, beams of electrons, protons or heavy ions) can induce changes in structure and/or chemical properties at the molecular level that might be lethal for living systems. Radiotherapy makes use of these harmful effects to target and destroy cells in risk zones or tumors. In this field, proton and carbon therapies have emerged as alternatives to traditional X-Ray treatments, since swift ions can deposit maximum energy at specific body depths (effect known as the 'Bragg peak'). Their high efficiency at lower doses gives rise to less aggressive secondary effects in normal tissues $[1,2]$. It is well established that the biological damage caused by these ions is the result of attacks to DNA molecules, usually classified as direct or indirect effects [3]. Direct effects occur when the radiation directly interacts with DNA molecules, while indirect effects refer to the interaction with the surrounding media, generally water radiolysis products. The understanding of such effects and their hierarchy on time is matter of extreme importance for both cancer treatment and cancer diagnosis.

Early stages of damage after irradiation have been approached by collisions experiments of DNA and RNA building blocks in gas phase [4-6]. Among them, cutting-edge multicoincidence detection techniques provide correlation between different charged fragments generated in single event collisions, allowing to determine which fragmentation channels are associated with a given biomolecule and not with the environment (see for instance Ref. [7]). In such experiments, fragmentation results from the relaxation of the excess of electronic energy associated with vacancies created in the different electronic shells of the molecule by the collisional process. However, as the electronic vacancies can be produced in many of these shells, experimental evidence cannot establish the influence of the shape or energy of the molecular orbital (MO) in which the electron vacancies are created; or indicate the dependence on the neighboring functional groups (the so-called intramolecular environment).

So far, very few theoretical works have focused on these primary processes leading to DNA or RNA bases fragmentation. Most of the quantum chemistry calculations exploring the mechanisms of biological damage have mainly studied singly ionized radicals of the bases in gas phase. These calculations have characterized geometries and energies, mostly for the purpose of assessing ionization potentials or to identify the most stable fragmentation pathway in the ground state. Reviews on these calculations can be found in Refs. [8-13]. Chemical reactivity of $\mathrm{OH}$ species or specific processes like hydrogen abstraction on DNA bases in gas phase have been also studied $[14,15]$.
All these findings enhance the importance of theoretical works to help obtaining a comprehensive view of the physics and chemistry of the primary events. However, the ionization process by itself and the subsequent chemical processes occurring at the femtosecond time-scale involve changes in the electronic states that cannot be captured within the Born-Oppenheimer (BO) approximation. Indeed, the recent development of molecular dynamics (MD) combined with time-dependent density functional theory (TDDFT) [16] in the Ehrenfest formalism [17, 18], made accessible the study of nonadiabatic effects like those occurring in collisions of ions with small molecules [19], radiolysis of bulk water [20] and collisions of swift heavy ions with small water clusters in gas phase [21].

In this article we use a combination of Ehrenfest-based TDDFT MD and BO MD simulations to study the early stages that follow the double ionization of uracil in gas phase. The theoretical outcome is compared to that of the recently published $100 \mathrm{keV}$ proton collision [22]. The aim of this work can be divided into two main interests: we want to check the accuracy of our theoretical approach and we also want to provide dynamical information that experiments alone can not provide (e.g. effects of the chemical environment). The choice of uracil corresponds to the large tradition within the collisional physics community in which it has been chosen as a benchmark because of its biological relevance (RNA base) and high thermal stability $[4,6,23-27]$. The paper has been divided as follows: Section 2 describes the theoretical methods used and also provides a brief survey of the proton collisional experiment, Section 3 discusses the main results and Section 4 summarizes the most important conclusions.

\section{Methodology and working hy- pothesis}

Our aim is to predict the fragmentation pathways once the uracil is in a doubly ionized excited state, therefore, the description of the collisional process (swift ion - biomolecule interaction) is out of reach of the model. To describe the electronic relaxation process we use the Ehrenfest-based TDDFT MD scheme as it is implemented in the CPMD package ${ }^{1}$. For all simulations presented here we use a cubic box of size $L=19 \AA$ and a plane wave basis with a kinetic energy cutoff of $70 \mathrm{Ry}$. Core elec-

\footnotetext{
1 CPMD package, Copyright IBM Corp 1990-2011, Copyright MPI fuer Festkoer-perforschung Stuttgart (1997-2001)
} 
trons are replaced by pseudopotentials of the standard Troullier-Martins form [28] and exchange correlation energy is calculated using the Becke-Lee-Yang-Parr (BLYP) functional $[29,30]$. The initial configuration of uracil is taken from an equilibrated trajectory obtained from a CarParrinello (CP) MD [31] simulation of the neutral system at $350 \mathrm{~K}$. The excited state of the doubly charged species is achieved through the removal of two electrons from selected inner-shell molecular orbitals of the neutral configuration, i.e. we consider a vertical ionization process. It is worth mentioning that ionizations from different orbitals are unaffordable from a computational view point. The use of the spin-polarized model (i.e. allowing electrons of different spin to have different spatial densities) requires an increase of about $80 \%$ of the computational effort, as we double the number of states to simulate. The vertical ionization assumption can be considered as a consequence of the ultra-short time-scales of the collision process. In the linear energy transfer range of ions relevant to our work, experimental conditions are such that the collision time is about $0.1 \mathrm{fs}$ (see, for instance, Refs. [32, 33] for liquid water). In that range of velocities, capture cross sections are negligible, meaning that the charge state of the colliding protons remains unchanged. Moreover, ion projectiles have velocities high enough so that elastic collisions can be safely neglected. Consequently, the energy of these particles upon collision with uracil is released through the electronic excitation and ionization of the target biomolecule.

The electronic degrees of freedom of the initial perturbed density of the doubly charged uracil $\left(\mathrm{Ura}^{2+}\right)$ are propagated by numerical integration of the time-dependent Kohn-Sham (KS) equations [16]. We use an iterative Crank-Nicholson algorithm with a time step of $\delta \mathrm{t}=$ $0.00024 \mathrm{fs}$ combined with a two-step Runge-Kutta scheme to maintain order $\delta \mathrm{t}^{3}$ accuracy [18]. The small step of integration chosen assures the conservation of energy with a tolerance of $0.4 \mathrm{eV}$ during the trajectory. This energy increase has been tested not to bias the dynamics. The computational cost of the time-dependent simulations necessarily limits the trajectories to less than $100 \mathrm{fs}$. In order to complete the dissociation process, we switch to ground-state Born-Oppenheimer (BO) MD when timedependent dynamics becomes essentially adiabatic (i.e., when TDDFT MD and BO MD produce the same trajectory). For the ground state dynamics we use a longer step of integration, $\delta \mathrm{t}=0.024 \mathrm{fs}$. Finally, the atomic charges are evaluated on the trajectories using the near-grid algorithm based on Bader's atoms-in-molecules approach [34].

The particular case of the ionization from the highest occupied molecular orbital (HOMO) is assumed to occur in the ground state of the Ura ${ }^{2+}$ and, therefore, is entirely simulated using CP MD. The sampling of trajectories at $350 \mathrm{~K}$ extended up to 5 ps have shown no fragmentation due to the presence of dissociation barriers. In order to assure an efficient crossing, ionization from the $\mathrm{HOMO}$ presented in Section 3 corresponds to calculations at 2300 and 2800 $\mathrm{K}$ that lead to the equivalent fragmentation patterns, occurring both at $\approx 0.5$ ps.

The coincidence two-dimensional time-of-flight (2D TOF) spectrum reported here has been previously published in Ref. [22]. A detailed description of the experimental setup can be found in [35] and references therein. In short, a pulsed proton beam of $100 \mathrm{keV}$ and $2 \mathrm{~mm}$ diameter collides with an uracil gas jet produced by heating commercial powder at a temperature of $120-150^{\circ} \mathrm{C}$. Charged fragments produced in the collision are analyzed according to their mass/charge ratio (M/Z) using a time-of-flight mass spectrometer operating in second order space focusing with pulsed extraction. The fragments are detected by a high efficiency multichannel plate (MCP) equipped with a secondary electron repeller grid in front of the first MCP. The TOF of all fragments generated by one ion pulse is measured by a multistop time device and stored in an event-by- event mode.

\section{Results}

We generate five TDDFT MD initial ionization conditions by removing two electrons from the 9a', 10a', 11a', 17a' and 5a" molecular orbitals of neutral uracil (nomenclature taken from Ref. [36]). In our KS pseudopotential representation inner-shell orbitals 9a', 10a' and 11a' (hereafter $\mathrm{KS} 1, \mathrm{KS} 2$, and $\mathrm{KS} 3$ ) correspond to the three deepest molecular orbitals; 17a' (KS9) is an intermediate-energy orbital and 5a" is the HOMO. Their corresponding electron densities are shown in Figure 1 as well as their energies, namely: 27, 26, 24, 14 and $6 \mathrm{eV}$ for the KS1, KS2, KS3, KS9 and HOMO orbitals. This sampling allows us to investigate the influence of the energy and shape of the ionized molecular orbital on the fragmentation processes, and it also reveals the effect of the chemical environment on a particular bond. KS1 and KS2 orbitals are associated with localized $\sigma$-like $\mathrm{C} 2=\mathrm{O}$ and $\mathrm{C} 4=\mathrm{O}$ bonds in a different intramolecular chemical environment: while KS1 is located between two $\mathrm{N}$ atoms, KS2 lies between $\mathrm{N}$ and $\mathrm{C}$ atoms. $\mathrm{KS} 3$ is delocalized over the $\mathrm{C} 2=\mathrm{O}$ bond and part of the uracil ring, and KS9 and $\mathrm{HOMO}$ orbitals are even more delocalized over the molecule. The rightside of Figure 1 shows the final stage of the dynamics after the removal of two electrons from the aforementioned orbitals. For each of the fragments mass/charge ratios 
and fragment-fragment distances are provided. The values of the charges must be taken with caution since the mean field potential energy surface that drives the molecular motion in the Ehrenfest approach is an average over potentially different (in ionic character) potential energy surfaces [37]. Besides, it is well-known that DFT-based methods are not able to properly describe charges of dissociation processes due to approximated exchange and correlation functionals [38]. Alternatively, two fragments with fractional charges can also arise from the combination of two dissociative electronic states driving the system along the same trajectory in configuration space but having different charge distributions (integer charges differently distributed on the fragments).

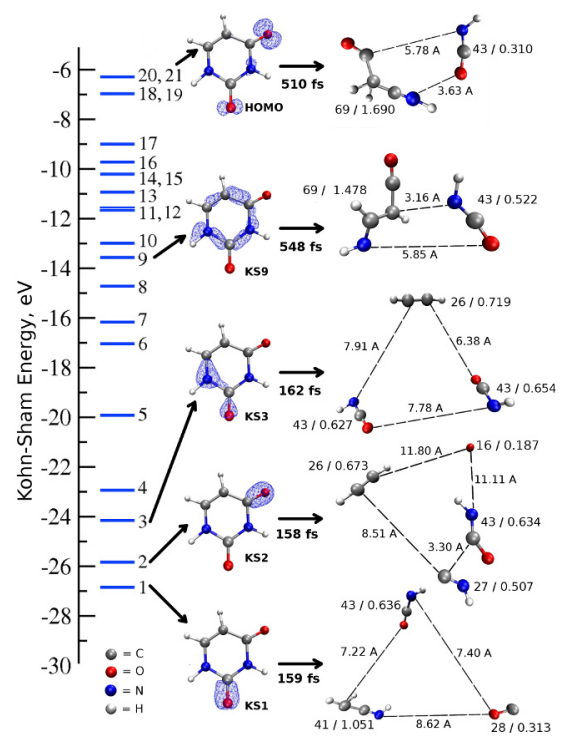

Figure 1. Left: Energies of the molecular orbitals of neutral uracil calculated in the pseudopotential approximation provided by the CPMD package. Centre: Electronic isodensities of the HOMO, KS9, KS3, KS2, and KS1 molecular orbitals (0.018 isovalue). Right: Fragments obtained at the end of the TDDFT and BO MD simulations initiated by a twoelectron removal from the selected orbital. $M / Z$ ratios and fragment-fragment distances (in $\AA$ ) are provided for each case.

The MD simulation starting from the deepest orbital ionization, KS1, leads to three fragments of masses 28, 41 and 43. The fragment of mass 41 has a charge of about +1 during the whole trajectory. The rest of the charge is shared between fragments of masses 28 and 43 that come from a common fragment of mass 71. Fractional charges suggest the formation of a singly charged fragment with mass 28 and a neutral fragment with mass 43 , and vice versa. Thus, this trajectory is compatible with 28/41 and 41/43 coincidences signals (where the notation means: light-fragment-mass/heavy-fragment-mass), and it might

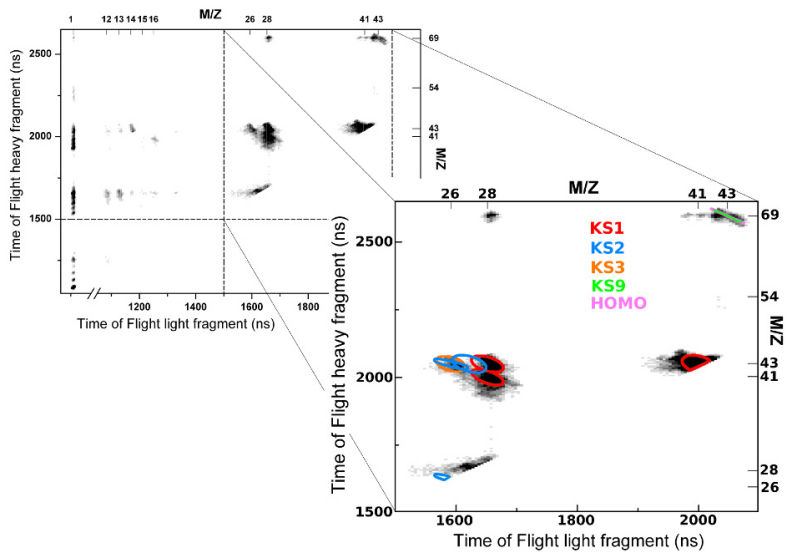

Figure 2. 2D TOF coincidence spectrum of charged uracil (data taken from Ref. [22]). In the inset, fragments for ratio $M / Z$ $\geq 22$ where the theoretical 2D TOF spectrum for KS1, KS2, $\mathrm{KS} 3, \mathrm{KS} 9$ and HOMO fragmentations is represented by colored symbols.

be also compatible with 28/43. The double ionization of the KS2 orbital instead leads to the most fragmented path in which one neutral $\mathrm{O}$ atom and three charged fragments are generated. The oxygen release is produced at the very first fs of the dynamics ( $\approx 6 \mathrm{fs}$ ), and involves the same $\mathrm{O}$ atom as the one taking part in the $\mathrm{CO}$ bond from which the two electron were initially removed. The rest of the molecule takes about $\approx 25$ fs to break into three fragments that share the $2+$ charge. Coincidence signals compatible with these results are 26/27, 26/43, and 27/43. None of these coincidences are seen in the KS1 fragmentation, showing that the chemical environment where the ionized orbitals are embedded plays a crucial role in the dynamics. For the case in which two electrons are removed from the KS3 orbital, one charged fragment of mass 26 and two charged fragments with mass 43 lead to an unique coincident signal of 26/43. Finally, ionizations from the KS9 and HOMO orbitals lead to a $43 / 69$ coincidence which is likely the case for two-electron vacancies created in any orbital between them. This statement is supported by additional calculations in which the initial internal energy of $\mathrm{Ura}^{2+}$ is set up to be equivalent to a double ionized species with two vacancies in the KS6-KS7 orbitals. For these additional cases we obtain very similar fragments that match the 43/69 signal of the KS9 and HOMO orbitals. This coincidence also corresponds to the most thermodynamically stable fragmentation path according to the all-electron calculations of Table 1. Thus, we can conclude that inner-shell vacancies favor dissociation channels that lie much higher in energy but are dynamically favored.

Figure 2 shows the 2D TOF ion/ion coincidence spectrum zoomed in to the signature of $\mathrm{Ura}^{2+}$ fragmentation. Combining the spectrum with the theoretical velocities for each 
Table 1. Energies for different fragmentation channels calculated at CCSD(T)/6-311++G(3df,2pd)//BLYP/6-311+G(d,p) level using Gaussian 03 package $^{a}$. Total energies of each fragmentation channel $(\mathrm{E}$, in $\mathrm{eV})$ are calculated by simple addition of the electronic energies of the single fragments. In the third column, these values are compared to that of Ura ${ }^{2+}\left(\mathrm{E}_{r e l}\right.$, in eV).

\begin{tabular}{|c|c|c|c|c|}
\hline & Fragments & Signal & $E(e V)$ & $\mathrm{E}_{\text {rel }}(\mathrm{eV})$ \\
\hline 0 & Uracil $^{2+}$ & - & -11257.94989 & - \\
\hline 1 & $\mathrm{HNCCH}_{2}^{1+}+\mathrm{OCNH}^{1+}+\mathrm{CO}$ & $41 / 43$ & -11246.77234 & 11.18 \\
\hline 2 & $\mathrm{HNCCH}_{2}^{1+}+\mathrm{OCNH}+\mathrm{CO}^{1+}$ & $41 / 28$ & -11244.36876 & 13.58 \\
\hline 3 & $\mathrm{HNCCH}_{2}+\mathrm{OCNH}^{1+}+\mathrm{CO}^{1+}$ & $43 / 28$ & -11241.53764 & 16.41 \\
\hline 4 & $\mathrm{HCCH}^{1+}+\mathrm{CNH}^{1+}+\mathrm{O}(\mathrm{NH}) \mathrm{C}+\mathrm{O}$ & $26 / 27$ & -11232.92048 & 25.03 \\
\hline 5 & $\mathrm{HCCH}^{1+}+\mathrm{CNH}+\mathrm{O}(\mathrm{NH}) \mathrm{C}^{1+}+\mathrm{O}$ & $26 / 43$ & -11233.80076 & 24.15 \\
\hline 6 & $\mathrm{HCCH}^{1+}+\mathrm{CNH}+\mathrm{O}(\mathrm{NH}) \mathrm{C}+\mathrm{O}^{1+}$ & $26 / 16$ & -11231.46252 & 26.49 \\
\hline 7 & $\mathrm{HCCH}+\mathrm{CNH}^{1+}+\mathrm{O}(\mathrm{NH}) \mathrm{C}^{1+}+\mathrm{O}$ & $27 / 43$ & -11233.16764 & 24.78 \\
\hline 8 & $\mathrm{HCCH}+\mathrm{CNH}^{1+}+\mathrm{O}(\mathrm{NH}) \mathrm{C}+\mathrm{O}^{1+}$ & $27 / 16$ & -11230.82940 & 27.12 \\
\hline 9 & $\mathrm{HCCH}+\mathrm{CNH}+\mathrm{O}(\mathrm{NH}) \mathrm{C}^{1+}+\mathrm{O}^{1+}$ & $43 / 16$ & -11231.70968 & 26.24 \\
\hline 10 & $\mathrm{C}_{2} \mathrm{H}_{2}^{1+}+\mathrm{OCNH}^{1+}+\mathrm{OCNH}$ & $26 / 43$ & -11243.72892 & 14.22 \\
\hline 11 & $\mathrm{C}_{2} \mathrm{H}_{2}+\mathrm{OCNH}^{1+}+\mathrm{OCNH}^{1+}$ & $43 / 43$ & -11243.50313 & 14.45 \\
\hline 12 & $\mathrm{HNC}_{2} \mathrm{H}_{2} \mathrm{CO}^{1+}+\mathrm{OCNH}^{1+}$ & $69 / 43$ & -11258.20567 & -0.26 \\
\hline 13 & $\mathrm{HNC}_{2} \mathrm{H}_{2} \mathrm{CO}^{2+}+\mathrm{OCNH}$ & - & -11244.60809 & 13.34 \\
\hline 14 & $\mathrm{HCCH}^{1+}+\mathrm{CO}^{1+}+\mathrm{OCNH}+\mathrm{NH}$ & $26 / 28$ & -11237.55032 & 20.40 \\
\hline 15 & $\mathrm{HCCH}^{1+}+\mathrm{CO}+\mathrm{OCNH}^{1+}+\mathrm{NH}$ & $26 / 43$ & -11239.95389 & 18.00 \\
\hline 16 & $\mathrm{HCCH}^{1+}+\mathrm{CO}+\mathrm{OCNH}+\mathrm{NH}^{1+}$ & $26 / 15$ & -11238.06003 & 19.89 \\
\hline 17 & $\mathrm{HCCH}+\mathrm{CO}^{1+}+\mathrm{OCNH}^{1+}+\mathrm{NH}$ & $28 / 43$ & -11237.32454 & 20.63 \\
\hline 18 & $\mathrm{HCCH}+\mathrm{CO}^{1+}+\mathrm{OCNH}+\mathrm{NH}^{1+}$ & 28/15 & -11235.43068 & 22.52 \\
\hline 19 & $\mathrm{HCCH}+\mathrm{CO}+\mathrm{OCNH}^{1+}+\mathrm{NH}^{1+}$ & $43 / 15$ & -11237.83425 & 20.12 \\
\hline 20 & $\mathrm{HNC}_{3} \mathrm{H}_{2}^{1+}+\mathrm{OCNH}^{1+}+\mathrm{O}$ & $53 / 43$ & -11241.50292 & 16.45 \\
\hline 21 & $\mathrm{HNC}_{3} \mathrm{H}_{2}^{1+}+\mathrm{OCNH}+\mathrm{O}^{1+}$ & $53 / 16$ & -11239.63762 & 18.31 \\
\hline 22 & $\mathrm{HNC}_{3} \mathrm{H}_{2}+\mathrm{OCNH}^{1+}+\mathrm{O}^{1+}$ & $43 / 16$ & -11236.69055 & 21.26 \\
\hline 23 & $\mathrm{HNCCH}_{2} \mathrm{CO}^{1+}+\mathrm{CNH}^{1+}+\mathrm{O}$ & $69 / 27$ & -11240.50512 & 17.44 \\
\hline 24 & $\mathrm{HNCCH}_{2} \mathrm{CO}^{1+}+\mathrm{CNH}+\mathrm{O}^{1+}$ & $69 / 16$ & -11239.04716 & 18.90 \\
\hline
\end{tabular}

${ }^{a}$ M. J. Frisch, G. W. Trucks, H. B. Schlegel et al., GAUSSIAN 03, Revision D.01, Gaussian, Inc., Wallingford, CT, (2004)

fragment predicted by the MD trajectories, we generate a theoretical 2D TOF spectrum that is superimposed using color symbols. In order to obtain this spectrum, all the momenta of charged fragments are extracted directly from the theoretical values and randomly rotated in space via Monte Carlo simulations. For each obtained distribution, only the component along the axis cell is kept giving a spot that, for the sake of clarity, is limited to colored circles in the figure. As can be seen, calculations predict the most intense coincidence signals, as well as their shape in the TOF distributions. This suggests that not only the fragments but also their relative velocities are correctly described by the theory.

\section{Conclusions}

Fragmentation of doubly charged uracil in gas phase studied by BOMD and Ehrenfest based TDDFT MD simula- tions shows a good agreement with results obtained in $100 \mathrm{keV}$ proton collision. Mass and charge ratios of the fragments predicted by theory, and also their relative velocities, are in accordance with the 2D TOF coincidence spectrum. Indeed, theory is able to provide the correct velocity distributions that match in position and shape with the experimental ones. Considering different initial electronic states of doubly charged uracil, we show that fragmentation depends critically on the energy, shape and intramolecular chemical environment of the ionized molecular orbital. Thus, ionizations from orbitals of similar energy and/or localized in similar bonds lead to very different fragmentation patterns, that do not always correspond to the energetically most favorable ones. Energy calculation of fragmentation patterns is, therefore, not sufficient to completely describe the dynamics of these coulombic driven processes.

The successful example studied in this work suggests that the same general approach may work for describing frag- 
mentations of more complex systems like nucleosides or nucleotides, that will reveal the relevance of specific ionization of ester and $\beta-\mathrm{N}_{1}$-glycosidic bonds. Furthermore, some of the present authors have extended the theoretical methods discussed here to study the fragmentation of uracil in aqueous solution [39]. This work highlights the active role of the solvent in the fragmentation process and represents a step forward in the theoretical description of more realistic biological environments.

\section{Acknowledgments}

This paper was intended for the special issue associated to the annual meeting of the COST Action CM0702 held in Cluj-Napoca, Romania between 21-23 March 2012. This work was performed using HPC resources from Mare Nostrum BSC, CCC-UAM, CCR UPMC and GENCI CINES/IDRIS grant 2010-085014. We thank the support of the MICINN projects FIS2010-15127, CTQ201017006, ACI2008-0777, and CSD 2007-00010, the CAM project S2009/MAT1726, the COST Action CM0702, the Picasso project HF2007-0067, and HPC Europa2. P. López-Tarifa, I. Tavernelli and U. Röthlisberger also acknowledge the Swiss NSF through the NCCR MUST interdisciplinary research program. P. López-Tarifa also thanks the FP7 Marie Curie COFUND Action.

\section{References}

[1] S. Braccini, Nucl. Phys. B 172, 8 (2007)

[2] H. Tsujii et al., J. Radiat. Res. 48, A1-A13 (2007)

[3] J. Ward, Prog. Nucleic Acid Res. Mol. Biol. 35, 95 (1988)

[4] J. de Vries et al., Phys. Rev. Lett. 91, 053401 (2003)

[5] B. Liu et al., Phys. Rev. Lett. 97, 133401 (2006)

[6] J. Tabet et al., Phys. Rev. A 81, 012711 (2010)

[7] J.-P. Champeaux et al., J. Phys. B: At. Mol. Opt. Phys. (2011)

[8] F. Ban, K. Rankin, J. Gauld, R. Boyd, Theor. Chem. Acc. 108, 11 (2002)

[9] S. Naumov, C. von Sonntag, Radiat. Res. 169, 355 (2008)

[10] N. Jena, P. C. Mishra, J. Phys. Chem. B. 109, 14205 (2005)

[11] A. Kumar, M. Sevilla, Radiation effects on DNA: theoretical investigations of electron hole and excitation pathways to DNA damage (Springer-Verlag, Berlin, 2008)
[12] X. Li, M. Sevilla, Adv. Quant. Chem. 52, 59 (2007)

[13] R. H. D. Lyngdoh et al., Acc. Chem. Res. 42, 563 (2009)

[14] C. J. Mundy, M. E. Colvin, A. A. Quong, J. Phys. Chem. A 106, 10063 (2002)

[15] Y. Wu, C. Mundy, M. Colvin, R. Car, J. Phys. Chem. A 108, 2922 (2004)

[16] E. Runge, E. K. U. Gross, Phys. Rev. Lett. 52, 997 (1984)

[17] A. Castro, M. A. L. Marques, J. A. Alonso, G. F. Bertsch, A. Rubio, Eur. Phys. J. D 28, 211 (2004)

[18] I. Tavernelli, U. F. Rohrig, U. Rothlisberger, Mol. Phys. 103, 963 (2005)

[19] G. Avendaño-Franco, B. Piraux, M. Grüning, X. Gonze, Theor. Chem. Acc. 131, 1289 (2012)

[20] I. Tavernelli et al., Chem. Phys. Chem. 9, 2099 (2008)

[21] L. Adoui et al. J. Phys. B: At. Mol. Opt. Phys. (2009)

[22] P. López-Tarifa et al., Phys. Rev. Lett. 107, 023202 (2011)

[23] B. Coupier et al., Eur. Phys. J. D 20, 459 (2002)

[24] J. de Vries et al., J. Phys. B 35, 4373 (2002)

[25] J. de Vries, R. Hoekstra, R. Morgenstern, T. Schlathoelter, Phys. Scr. T110, 336 (2004)

[26] T. Schlatholter, R. Hoekstra, R. Morgenstern, Int. J. Mass Spectrom. 233, 173 (2004)

[27] P. Moretto-Capelle, A. Le Padellec, Phys. Rev. A 74, 062705 (2006)

[28] N. Troullier, J. L. Martins, Phys. Rev. B 43, 1993 (1991)

[29] A. D. Becke, Phys. Rev. A 38, 3098 (1988)

[30] C. Lee, W. Yang, R. G. Parr, Phys. Rev. B 37, 785 (1988)

[31] R. Car, M. Parrinello, Phys. Rev. Lett. 22, 2471 (1985)

[32] M. P. Gaigeot et al., J. Phys. B: At. Mol. Opt. Phys. 40, 1 (2007)

[33] I. Tavernelli, M. P. Gaigeot, R. Vuilleumier, C. Stia, M. A. Hervé du Penhoat, M. F. Politis, Chem. Phys. Chem. 9, 2099 (2008)

[34] W. Tang, E. Sanville, G. Henkelman, J. Phys.: Comput. Mater. 21, 084204 (2009)

[35] P. Cafarelli et al., AIP Conf. Proc. 1080, 71 (2008)

[36] D. M. P. Holland, A. W. Potts, L. Karlsson, I. L. Zaytseva, A. B. Trofimov, J. Schirmer, Chem. Phys. 353, 47 (2008)

[37] N. L. Doltsinis, D. Marx, J. Theor. Comp. Chem. 1, 319 (2002)

[38] A. J. Cohen, P. Mori-Sánchez, W. Yang, Science 321, 792 (2008)

[39] P. López-Tarifa et al., Angew. Chem. 52, 3160 (2013) 